\title{
Prognostic significance of combined preoperative platelet-to-lymphocyte ratio and lymphocyte-to- monocyte ratio in patients undergoing surgery with stage IB non-small-cell lung cancer
}

This article was published in the following Dove Press journal:

Cancer Management and Research

\author{
Yongqiang Chen ${ }^{1, *}$ \\ Weidong Wang ${ }^{1, *}$ \\ Xuewen Zhang ${ }^{2}$ \\ Xiangyang $\mathrm{Yu}^{3}$ \\ Kexing $\mathrm{Xi}^{4}$ \\ Yingsheng Wen' \\ Gongming Wang' \\ Xiaoli Feng' \\ Lanjun Zhang'
}

'Department of Thoracic Surgery, Sun Yat-Sen University Cancer Center, Guangzhou, Guangdong, China; ${ }^{2}$ Department of Medical Oncology,

Sun Yat-Sen University Cancer

Center, Guangzhou, Guangdong,

China; ${ }^{3}$ Department of Thoracic Surgical Oncology, Cancer Institute and Hospital, Chinese Academy of Medical Sciences, National Cancer Center, Beijing, China; ${ }^{4}$ Department of Thoracic Surgery, The First Affiliated Hospital of Jinan University, Guangzhou, Guangdong, China

*These authors contributed equally to this work
Correspondence: Lanjun Zhang Department of Thoracic Surgery, Sun Yat-sen University Cancer Center, 65I Dong Feng east Road, 510060 guangzhou,

guangdong, China

Tel +862087343258

Fax +86 2087343258

Email zhanglj@sysucc.org.cn
Background: Research indicates that the presence of a systemic inflammatory response plays an important role in predicting survival in patients with cancer. The aim of this study was to investigate the prognostic value of preoperative neutrophil-to-lymphocyte ratio (NLR), lymphocyte-to-monocyte ratio (LMR), platelet-to-lymphocyte ratio (PLR), prognostic nutritional index, and the combination of preoperative LMR and PLR (LMR-PLR) in predicting the survival of patients with stage IB non-small-cell lung cancer (NSCLC).

Materials and methods: We retrospectively analyzed clinical data of 577 patients with stage IB NSCLC who underwent pneumonectomy from January 1999 to December 2009. Univariate and multivariate Cox survival analyses were used to evaluate the prognostic indicators, including LMR-PLR. The cutoff values for LMR and PLR were defined by the receiver operating characteristic (ROC) curve analysis. According to the ROC curve, the recommended cutoff values of LMR and PLR were 3.16 and 81.07, respectively. We divided the patients into three groups according to their LMR and PLR status and defined them with different scores. Patients with both high LMR (>3.16) and low PLR $(\leq 81.07)$ were given a score of 2, whereas those with one or neither were scored 1 or 0 , respectively. Survival curves were plotted using the Kaplan-Meier method and compared with the log-rank test. Cox proportional hazards analyses were used to identify the factors associated with overall survival (OS).

Results: The median follow-up time was 93.77 months. The allocation of the LMR-PLR score was as follows: LMR-PLR = 0, 193 (33.4\%) patients; LMR-PLR = 1, 308 (53.4\%) patients; and LMR-PLR $=2,76(13.2 \%)$ patients. After multivariate analysis, our results showed that LMR-PLR was an independent prognostic indicator for OS $(P=0.001)$. The 10 -year OS rates were $70.0 \%$, $60.4 \%$, and $49.5 \%$ for LMR-PLR $=2$, LMR-PLR $=1$, and LMR-PLR $=0$, respectively $(P<0.001)$. Conclusion: This study demonstrated that preoperative LMR and PLR are simple, readily available, and low-cost biomarkers. Preoperative LMR-PLR score can be used as a valuable prognostic marker for long-term survival in stage IB NSCLC patients who underwent surgery. Keywords: IB non-small-cell lung cancer, platelet-to-lymphocyte ratio, lymphocyte-to-monocyte ratio, prognostic factors, systemic inflammation, pneumonectomy

\section{Introduction}

Non-small-cell lung cancer (NSCLC), accounting for $\sim 85 \%$ of all lung cancers, has become a leading cause of cancer-related mortality and morbidity worldwide. According to the latest world cancer epidemiology statistics report, it is the most common malignant tumor and is accountable for the highest cancer mortality rates in the world for men and women in developed countries. ${ }^{1,2}$ 
In the past few years, many early or asymptomatic lung cancers have been detected with cancer screening conducted by the Chinese cancer society in areas with high lung cancer incidence. ${ }^{3}$ Although NSCLC therapy has recently seen substantial progress, the diagnostic accuracy of this condition in the early stages remains unsatisfactory and the 5-year overall survival (OS) rate of NSCLC ranges from $15 \%$ to $35.9 \%$ even after radical resection of lung cancer., ${ }^{4,5}$ Therefore, it is necessary to identify prognostic factors for predicting stage IB NSCLC. At the same time, precise prognosis in patients is very important to ensure that the best treatment plan and follow-up strategy are selected. In recent years, the theory of inflammatory reactions involved in the occurrence and development of various malignant solid tumors has been widely studied and has become a potential and promising prognostic indicator. In addition, as most stage IB patients with NSCLC have long-term survival, only tumor diameter, low-differentiated tumor, angiolymphatic invasions, pleural involvement, and bronchial infiltration have been found to be independent prognostic indicators for the postoperative OS reported. ${ }^{6,7}$ Studies so far have found that neutrophilto-lymphocyte ratio (NLR), lymphocyte-to-monocyte ratio (LMR), platelet-to-lymphocyte ratio (PLR), and prognostic nutritional index (PNI) are good inflammatory response follow-up markers and predictive survival markers in patients with different carcinomas. ${ }^{8-10}$ However, only a few studies have reported results in this regard in early NSCLC, especially the IB period. For patients with stage IB NSCLC, we can use cranial magnetic resonance imaging (MRI) and chest and abdomen enhancement computed tomography (CT) scans to assess the patient's clinical status and disease severity. However, these tests are both expensive and inconvenient. Therefore, it is necessary to establish other simple, inexpensive, and promising prognostic factors for stage IB NSCLC. More importantly, they can be easily obtained in everyday practice, without additional costs. ${ }^{11,12}$ Recently, in an aim to further refine prognostication, hematological indices have been variably combined to generate a number of prognostic inflammatory scores, including the combination of platelet count and NLR and the combination of LMR and PLR (LMR-PLR) which were developed and shown to be independently associated with shortened survival in several tumor types. ${ }^{13-16}$ Moreover, the prognostic role of LMR-PLR in stage IB NSCLC in terms of survival outcomes has not been studied. ${ }^{14}$

Therefore, in this study, we aimed to explore the prognostic value of these biomarkers in patients with stage IB NSCLC.

\section{Materials and methods}

\section{Patients}

We retrospectively examined data from consecutive patients with NSCLC who underwent radical resection of lung cancer at Sun Yat-Sen University Cancer Center from January 1999 to December 2009. Patient demographics, tumor characteristics, preoperative treatment, and follow-up results were reviewed in detail from the medical records and the follow-up system. The present study was undertaken according to ethical standards of the World Medical Association Declaration of Helsinki. Approval was obtained from the Institute Research Medical Ethics Committee of Sun Yat-Sen University Cancer Center (no B2018-011), and written informed consent was obtained from all patients prior to the use of these sera. The enrolled patients met the following inclusion criteria: 1) no neoadjuvant treatment before operation; 2) surgical resection of lung lobectomy; 3 ) postoperative diagnosis of T2a stage NSCLC established by two board-certified pathologists independently; and 4) all lymph nodes confirmed negative (N0). Patients were excluded based on following criteria: 1) missing data on the number of blood cells before lung resection; 2) accompaniment with preoperative infection or other bone marrow, hematological, or autoimmune disease; and 3) loss to follow-up. A total of 598 patients was diagnosed with stage IB NSCLC. According to the exclusion criteria, 577 patients were finally enrolled in this study.

\section{Surgery and postoperative chemotherapy}

Preoperative preparation of patients selected for surgery included cardiac ultrasound, pulmonary function assessment, CT, and MRI. Tumor stage was classified according to the seventh edition of the American National Comprehensive Cancer Network for NSCLC. The clinician, based on the size and location of the tumor, chose lung cancer radical mastectomy (pulmonary lobectomy and node dissection). Standard systematic lymph node dissection requires at least six groups, including three intrapulmonary lymph nodes and three mediastinal lymph nodes. According to the postoperative pathological results, clinicians recommended postoperative adjuvant chemotherapy for patients with high-risk recurrence factors. High-risk factors for recurrence included: 1) tumor diameter $>4 \mathrm{~cm}$ and 2 ) invasion of visceral pleura or bronchus. Postoperative adjuvant chemotherapy is generally recommended 4-6 weeks after surgery and includes platinum-containing drugs pemetrexed, docetaxel, gemcitabine, vinorelbine, and paclitaxel. 


\section{Inflammatory index calculation}

Systemic inflammatory data represent neutrophil, lymphocyte, monocyte, and platelet counts that were obtained from routine blood tests performed a week prior to the surgery using Sysmex XE-5000 ${ }^{\mathrm{TM}}$ Automated Hematology System (Sysmex Corporation, Kobe, Japan). PLR was defined as the ratio of the platelet count to the lymphocyte count, LMR was the ratio of the lymphocyte count to the monocyte count, NLR was the ratio of the neutrophil count to the lymphocyte count, and the PNI was calculated as $10 \times$ serum albumin value $(\mathrm{g} / \mathrm{dL})+0.005 \times$ peripheral lymphocyte count $\left(\right.$ per $\left.\mathrm{mm}^{3}\right)$.

\section{Follow-up}

Follow-up observations commenced from the date of radical resection. Follow-up visits were held every 3 months for the first 2 years after the completion of surgery, every 6 months for the third through fifth years, and then once every year thereafter. Evaluations at each visit included complete blood count, biochemical examination, common tumor markers, chest radiography, and upper abdominal ultrasonography. In addition, chest and abdomen enhancement CT scans were performed every 6 months for the first 3 years and then annually until death. Brain MRI was performed annually. OS was calculated from the date of lobectomy to the date of death from any cause and censored at the time of the last follow-up visit for patients still alive. All follow-up statistics were reviewed by May 1, 2018.

\section{Statistical analysis}

All statistical analyses were completed using SPSS 20.0 (IBM Corporation, Armonk, NY, USA). All the performed tests were two-sided. $P$-values $<0.05$ were considered significant. First, the best cutoff values of these inflammation-based prognostic scores were determined by the maximum Youden index, which was generated by the respective receiver operating characteristic (ROC) curves, to evaluate the predictive values in prognosis. In addition, differences in categorical variables between groups were studied using chi-squared test. Needless to say, the prognostic factors of OS were confirmed by Kaplan-Meier univariate analysis using log-rank test. Significantly prognostic variables $(P<0.050)$ obtained from the Kaplan-Meier analysis were enrolled in Cox multivariate regression analysis based on the forward stepwise method.

\section{Results \\ Clinicopathological characteristics of patients}

All patients underwent surgery for stage IB NSCLC. The baseline clinicopathological characteristics of 577 patients with stage IB NSCLC are summarized in Table 1. There were $410(71.1 \%)$ males and $167(28.9 \%)$ females, the median age was 60 years (range 23-87 years). Meanwhile, 339 (58.8\%) patients had smoking history. After complete resection, 331 (57.4\%) patients had adenocarcinoma, 193 (33.4\%) patients had squamous cell carcinoma, and $71(19.8 \%)$ patients had other histologies. Approximately 39\% of the patients had visceral pleura invasion and $25.8 \%$ of the patients had bronchial invasion. Furthermore, the median and average count of resected lymph nodes (RLNs) were 13.0 and 14.1, respectively (interquartile range 8-19), and the median and average stations of RLNs were 5.0 and 4.5 , respectively (interquartile range 3-6) (Table 1).

\section{Best cutoff values for NLR, LMR, PLR, $\mathrm{PNI}$, and LMR-PLR definition}

At baseline, the median values of NLR, LMR, PLR, and PNI for the study population were 2.09 (range 0.11-19.25), 3.67 (range 1.14-57.67), 116.49 (range 30.73-626), and 52.40 (range 11.70-76.80), respectively. The maximum Youden indexes for these measures were $0.080,0.150$, 0.879 , and 0.098 . The optimal cutoff values for the prediction of OS by ROC analysis were 3.13 for NLR, 3.16 for LMR, 81.07 for PLR, and 49.55 for PNI (Figure 1). Consequently, patients were separately divided into two groups of high or low levels according to the optimal

Table I Clinicopathologic features of patients involved in this study

\begin{tabular}{|l|l|l|}
\hline Characteristics & & $\begin{array}{l}\text { Total } \\
\mathbf{n = 5 7 7}(\%)\end{array}$ \\
\hline Age (years) & $\leq 60$ & $297(51.5)$ \\
Sex & $>60$ & $280(48.5)$ \\
Smoking & Female & $167(28.9)$ \\
& Male & $410(71.1)$ \\
Cell types & Yes & $339(58.8)$ \\
& No & $238(41.2)$ \\
Tumor differentiation & Adenocarcinoma & $331(57.4)$ \\
Visceral pleura invasion & Squamous & $193(33.4)$ \\
& Others & $53(9.2)$ \\
Bronchial invasion & Yoorly or undifferentiated & $347(60.1)$ \\
& No & $230(39.9)$ \\
Postoperative & Yes & $225(39.0)$ \\
chemotherapy & No & $352(61.0)$ \\
Resected lymph node & Yes & $149(25.8)$ \\
numbers & No & $428(74.2)$ \\
Resected lymph node & $<12$ & $94(16.3)$ \\
stations & $\geq 12$ & $483(83.7)$ \\
& $<6$ & $255(44.2)$ \\
& $\geq 6$ & $322(55.8)$ \\
& & $413(71.6)$ \\
& & $164(28.4)$ \\
\hline
\end{tabular}


cutoff values. Moreover, 448 patients $(77.6 \%)$ had NLR $\leq 3.13,207$ patients $(35.9 \%$ ) had LMR $\leq 3.16,90$ patients $(15.6 \%)$ had PLR $\leq 81.07$, and 190 patients $(32.9 \%)$ had PNI $\leq 49.55$ (Table 2).

Based on these cutoff values, we calculated the LMRPLR score. Patients with both high LMR (>3.16) and low PLR $(\leq 81.07)$ were given a score of 2 . Patients with either high LMR $(>3.16)$ or low PLR $(\leq 81.07)$ were given a score of 1 . Patients without either abnormality were scored 0 . Table 3 illustrates the relationship of the clinicopathologic characteristics with patients grouped by their LMR-PLR score. The allocation of the LMR-PLR score was as follows:
LMR-PLR = 0, 193 (33.4\%) patients; LMR-PLR = 1, 308 $(53.4 \%)$ patients; and LMR-PLR $=2,76(13.2 \%)$ patients.

\section{Association of inflammation-based factors with clinicopathological characteristics}

The relationship between patient characteristics and NLR, LMR, PLR, and PNI is shown in Table 2. The high-LMR group and high-PLR group were significantly inclined to males $(P<0.001, \mathrm{P}=0.011)$, and patients with highLMR were significantly well or moderately differentiated $(P=0.003)$ than those with $\mathrm{LMR} \leq 3.16$. Moreover, patients who used tobacco also had higher PLR $(P=0.006)$. The
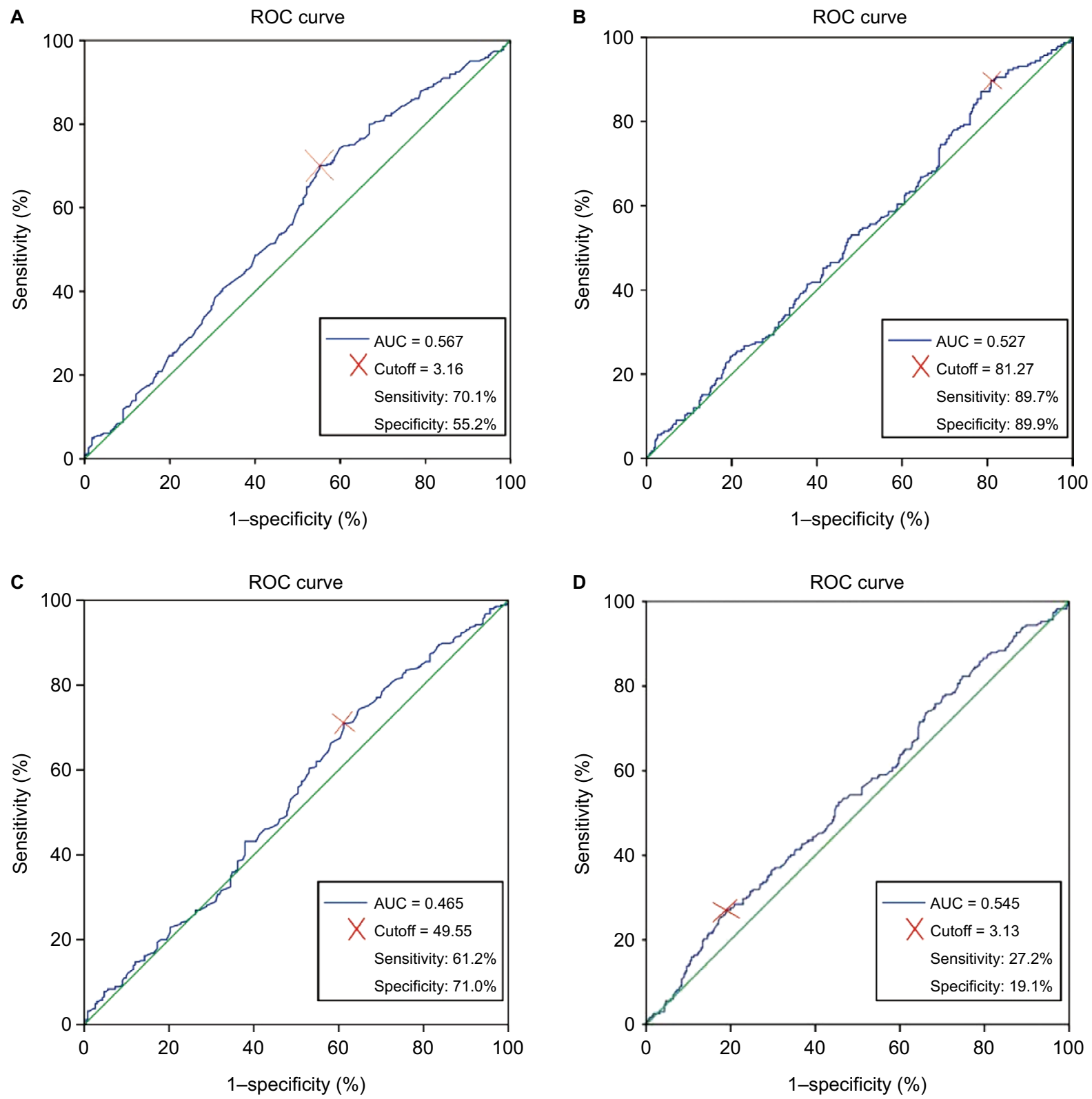

Figure I ROC curve for determination of the cutoff value for LMR (A), PLR (B), PNI (C), and NLR (D) in patients undergoing surgery with stage IB non-small-cell lung cancer. Abbreviations: AUC, area under the curve; LMR, lymphocyte-to-monocyte ratio; NLR, neutrophil-to-lymphocyte ratio; PLR, platelet-to-lymphocyte ratio; PNI, prognostic nutritional index; ROC, receiver operating characteristic. 


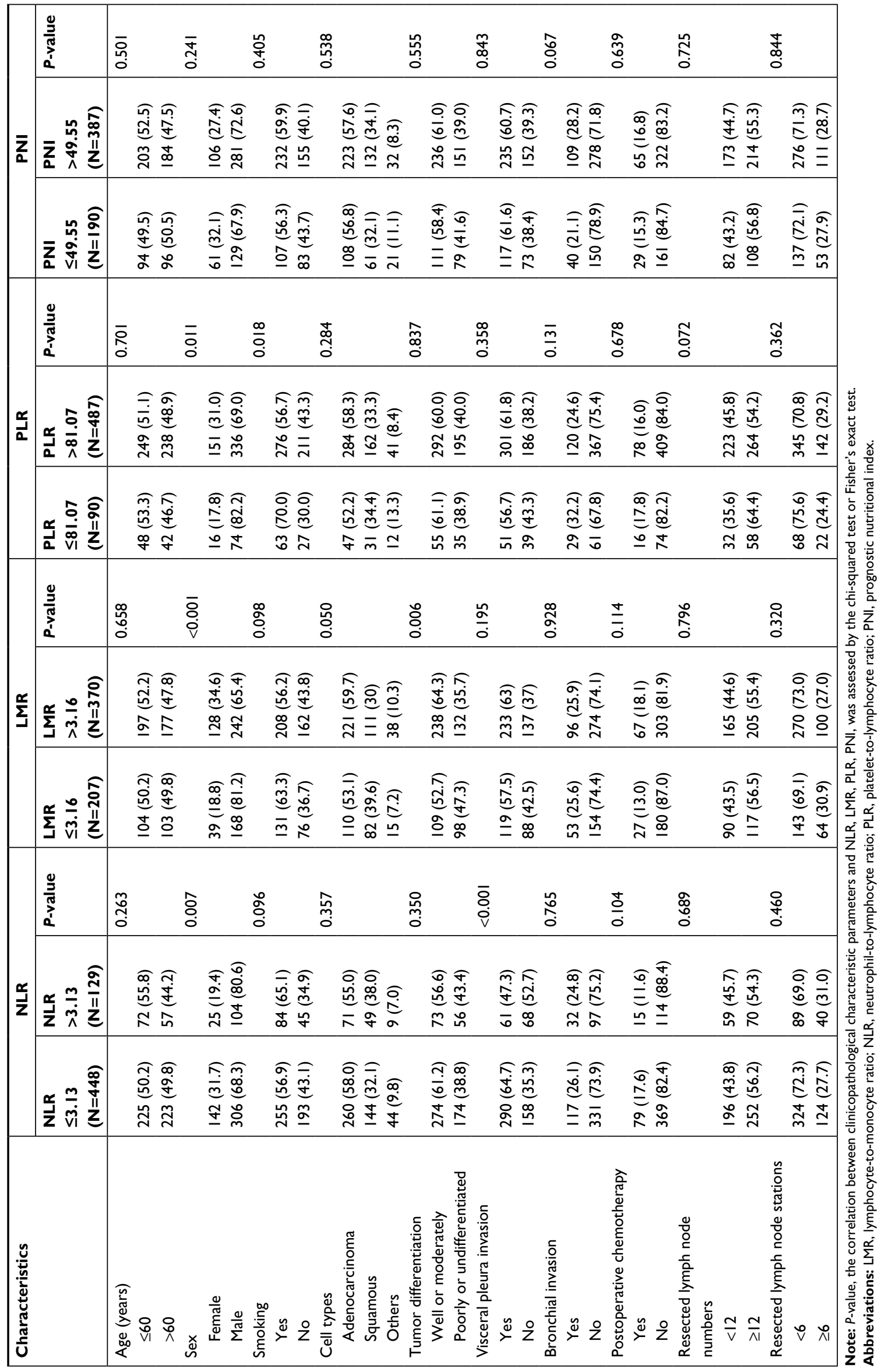


Table 3 Correlation between preoperative LMR-PLR and patient characteristics

\begin{tabular}{|c|c|c|c|c|}
\hline \multirow[t]{2}{*}{ Characteristics } & \multicolumn{3}{|c|}{ LMR-PLR score, n (\%) } & \multirow[t]{2}{*}{$P$-value } \\
\hline & 0 & 1 & 2 & \\
\hline Age (years) & & & & 0.777 \\
\hline$\leq 60$ & $98(50.8)$ & $157(51.0)$ & $42(55.3)$ & \\
\hline$>60$ & $95(49.2)$ & I5I (49.0) & $34(44.7)$ & \\
\hline Sex & & & & $<0.001$ \\
\hline Female & $39(20.2)$ & $112(36.4)$ & $16(21.1)$ & \\
\hline Male & I54 (79.8) & $196(63.6)$ & $60(78.9)$ & \\
\hline Smoking & & & & 0.092 \\
\hline Yes & $119(61.7)$ & $169(54.9)$ & $51(67.1)$ & \\
\hline No & $74(38.3)$ & $139(45.1)$ & 25 (32.9) & \\
\hline Cell types & & & & 0.043 \\
\hline Adenocarcinoma & $102(52.8)$ & $190(61.7)$ & $39(51.3)$ & \\
\hline Squamous & 77 (39.9) & $90(29.2)$ & $26(34.2)$ & \\
\hline Others & $14(7.3)$ & $28(9.1)$ & II (I4.5) & \\
\hline Tumor differentiation & & & & 0.040 \\
\hline Well or moderately & $102(52.8)$ & $197(64.0)$ & $48(63.2)$ & \\
\hline Poorly or undifferentiated & $91(47.2)$ & III (36.0) & $28(36.8)$ & \\
\hline Visceral pleura invasion & & & & 0.804 \\
\hline Yes & $114(59.1)$ & $191(62.0)$ & $46(60.5)$ & \\
\hline No & 79 (40.9) & $117(38.0)$ & $30(39.5)$ & \\
\hline Bronchial invasion & & & & 0.605 \\
\hline Yes & $47(24.4)$ & $79(25.6)$ & $23(30.3)$ & \\
\hline No & $146(75.6)$ & $229(74.4)$ & $53(69.7)$ & \\
\hline Postoperative chemotherapy & & & & 0.372 \\
\hline Yes & $26(13.5)$ & $53(17.2)$ & $15(19.7)$ & \\
\hline No & $167(86.5)$ & $255(82.8)$ & $61(80.3)$ & \\
\hline Resected lymph node numbers & & & & 0.370 \\
\hline$<12$ & $86(44.6)$ & |4| (45.8) & $28(36.8)$ & \\
\hline$\geq 12$ & $107(55.4)$ & $167(54.2)$ & $48(63.2)$ & \\
\hline Resected lymph node stations & & & & 0.461 \\
\hline$<6$ & $133(68.9)$ & $222(72.1)$ & $58(76.3)$ & \\
\hline$\geq 6$ & $60(31.1)$ & $86(27.9)$ & $18(23.7)$ & \\
\hline
\end{tabular}

Note: $P$-value, the correlation between clinicopathological characteristic parameters and preoperative LMR-PLR, was assessed by the chi-squared test or Fisher's exact test. Abbreviations: LMR, lymphocyte-to-monocyte ratio; LMR-PLR, combination of preoperative LMR and PLR; PLR, platelet-to-lymphocyte ratio.

association between LMR-PLR and clinicopathologic indexes of patients with NSCLC is shown in Table 3. We found significant correlation of LMR-PLR with sex $(P<0.001)$, smoking $(P=0.032)$, cell types $(P=0.043)$, and tumor differentiation $(P=0.040)$.

\section{Analysis of the prognostic values for OS}

The median follow-up time for the 577 patients was 93.77 (range 5.03-198.10) months. A total of 232 patients died during the observation period. The 1-, 3-, 5-, and 10-year OS rates were $96.3 \%, 84.3 \%, 71.9 \%$, and $58.0 \%$, respectively. In our study, only 94 (16.3\%) individuals received postoperative adjuvant chemotherapy. During the followup period, there were only 141 patients with postoperative recurrence of NSCLC including 67 (11.6\%) with distant metastasis and $74(12.8 \%)$ with local recurrences. Based on the cutoff values, we segregated the patients into dif- ferent groups. In the univariate analysis, we found that LMR, PLR, PNI, and LMR-PLR were correlated with OS ( $P=0.001, P=0.009, P=0.014$, and $P=0.001$, respectively). Five clinical characteristics were found to be significant (all $P<0.050)$ for OS by the univariate analysis: age, smoking status, cell type, bronchial invasion, and RLNs were also significant for OS.

As shown in Table 4, the multivariate analysis showed that LMR-PLR (HR, 0.434; 95\% CI, 0.265-0.710; $P=0.001$ ), age $>60$ years (HR, $1.701 ; 95 \% \mathrm{CI}, 1.304$ 20.218; $P<0.001$ ), smoking status (HR, $0.717 ; 95 \% \mathrm{CI}$, $0.542-0.950 ; \mathrm{P}=0.020$ ), and $\mathrm{RLN} \geq 12$ (HR, 0.736; 95\% CI, 0.567-0.956; $P=0.022$ ) were significant prognostic factors for the 10-year OS rate. Consequently, age, smoking status, cell type, and RLN were also revealed as independent prognostic factors (Table 4). The multivariate analysis also showed that LMR-PLR was considered 
Table 4 Univariate and multivariate analyses using Cox proportional hazards models using factors influencing OS

\begin{tabular}{|c|c|c|c|c|}
\hline \multirow[t]{3}{*}{ Characteristics } & \multicolumn{4}{|l|}{ OS } \\
\hline & \multirow{2}{*}{$\begin{array}{l}\begin{array}{l}\text { Univariate } \\
\text { analysis }\end{array} \\
\text { HR }(95 \% \mathrm{Cl})\end{array}$} & \multirow[t]{2}{*}{$P$-value } & \multirow{2}{*}{$\begin{array}{l}\begin{array}{l}\text { Multivariate } \\
\text { analysis }\end{array} \\
\text { HR (95\% CI) }\end{array}$} & \multirow[t]{2}{*}{$P$-value } \\
\hline & & & & \\
\hline \multicolumn{5}{|l|}{ Age (years) } \\
\hline$\leq 60$ & I (reference) & $<0.001$ & I (reference) & $<0.001$ \\
\hline$>60$ & $1.785(1.372-2.321)$ & & $1.701(1.304-2.218)$ & \\
\hline \multicolumn{5}{|l|}{ Sex } \\
\hline Male & I (reference) & 0.053 & & \\
\hline Female & $0.743(0.550-1.004)$ & & & \\
\hline \multicolumn{5}{|l|}{ Smoking } \\
\hline Yes & I (reference) & 0.033 & I (reference) & 0.020 \\
\hline No & $0.748(0.572-0.977)$ & & $0.717(0.542-0.950)$ & \\
\hline \multicolumn{5}{|l|}{ Cell types } \\
\hline Adenocarcinoma & I (reference) & 0.003 & I (reference) & 0.002 \\
\hline Squamous & $0.74 I(0.559-0.983)$ & & $0.687(0.510-0.925)$ & \\
\hline Others & $0.402(0.223-0.724)$ & & $0.429(0.237-0.775)$ & \\
\hline \multicolumn{5}{|l|}{ Tumor differentiation } \\
\hline Poorly or undifferentiated & I (reference) & 0.134 & & \\
\hline Well or moderately & $0.820(0.632-1.063)$ & & & \\
\hline \multicolumn{5}{|l|}{ Visceral pleura invasion } \\
\hline Yes & I (reference) & 0.403 & & \\
\hline No & $0.893(0.684-1.165)$ & & & \\
\hline \multicolumn{5}{|l|}{ Bronchial invasion } \\
\hline Yes & I (reference) & 0.024 & & \\
\hline No & $0.689(0.499-0.952)$ & & & \\
\hline \multicolumn{5}{|l|}{ Postoperative chemotherapy } \\
\hline Yes & I (reference) & 0.091 & & \\
\hline No & $1.392(0.948-2.044)$ & & & \\
\hline \multicolumn{5}{|l|}{ Resected lymph node numbers } \\
\hline$<12$ & I (reference) & 0.016 & I (reference) & 0.022 \\
\hline$\geq 12$ & $0.728(0.563-0.942)$ & & $0.736(0.567-0.956)$ & \\
\hline \multicolumn{5}{|l|}{ Resected lymph node stations } \\
\hline$<6$ & I (reference) & 0.910 & & \\
\hline$\geq 6$ & $0.984(0.742-1.305)$ & & & \\
\hline \multicolumn{5}{|l|}{ NLR } \\
\hline$\leq 3.13$ & I (reference) & 0.072 & & \\
\hline$>3.13$ & $1.354(0.974-1.884)$ & & & \\
\hline \multicolumn{5}{|l|}{ LMR } \\
\hline$\leq 3.16$ & I (reference) & 0.001 & & \\
\hline$>3.16$ & $0.647(0.499-0.839)$ & & & \\
\hline \multicolumn{5}{|l|}{ PLR } \\
\hline$\leq 81.07$ & I (reference) & 0.009 & & \\
\hline$>81.07$ & $1.757(1.151-2.682)$ & & & \\
\hline \multicolumn{5}{|l|}{ PNI } \\
\hline$\leq 49.55$ & I (reference) & 0.014 & & \\
\hline$>49.55$ & $0.717(0.55 I-0.934)$ & & & \\
\hline \multicolumn{5}{|l|}{ LMR-PLR } \\
\hline 0 & I (reference) & 0.001 & I (reference) & 0.001 \\
\hline I & $0.687(0.524-0.899)$ & & $0.685(0.523-0.898)$ & \\
\hline 2 & $0.426(0.261-0.934)$ & & $0.434(0.265-0.710)$ & \\
\hline
\end{tabular}

Note: $P$-value; in Cox hazards regression analysis, variables that were found to be statistically significant $(P<0.05)$ in the univariate analysis were entered into a Cox regression multivariate model using a forward conditional method.

Abbreviations: LMR, lymphocyte-to-monocyte ratio; LMR-PLR, combination of preoperative LMR and PLR; NLR, neutrophil-to-lymphocyte ratio; OS, overall survival; PLR, platelet-to-lymphocyte ratio; PNI, prognostic nutritional index. 
an independent prognostic indicator for OS in stage IB NSCLC after surgery.

\section{Patients' survival}

The Kaplan-Meier method was used to investigate the significance of age, smoking, cell types, RLNs, LMR, PLR, and LMR-PLR for predicting the OS of patients with NSCLC. Kaplan-Meier analysis indicated that the 10-year OS rate in the high-LMR group was significantly higher than that in

A

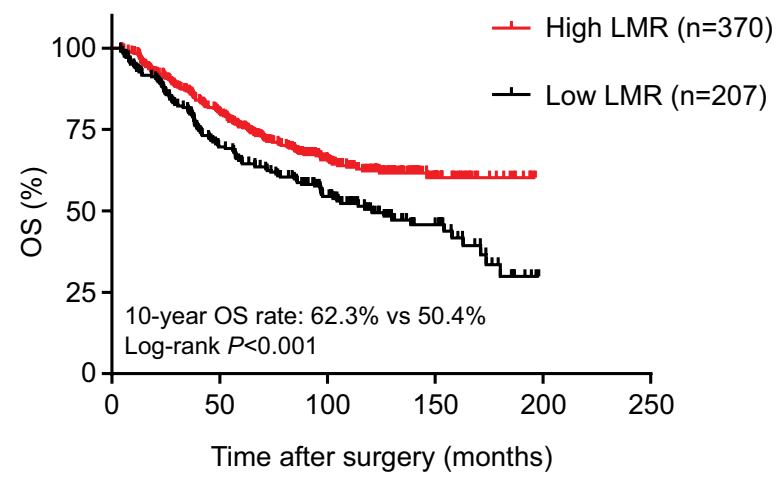

C

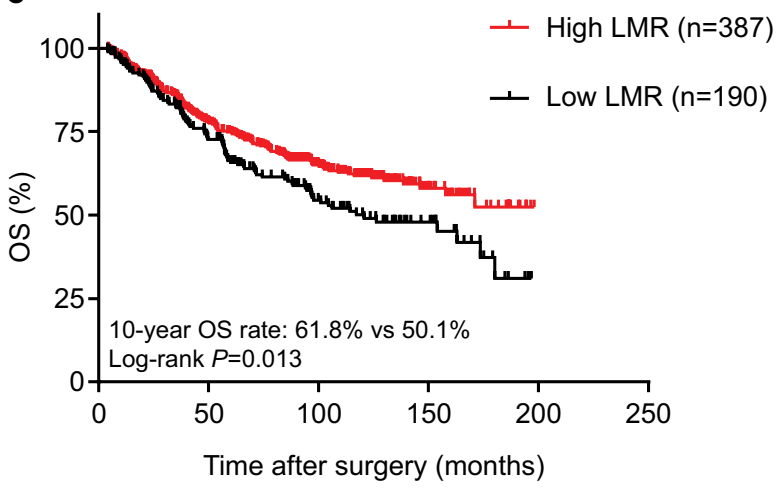

E

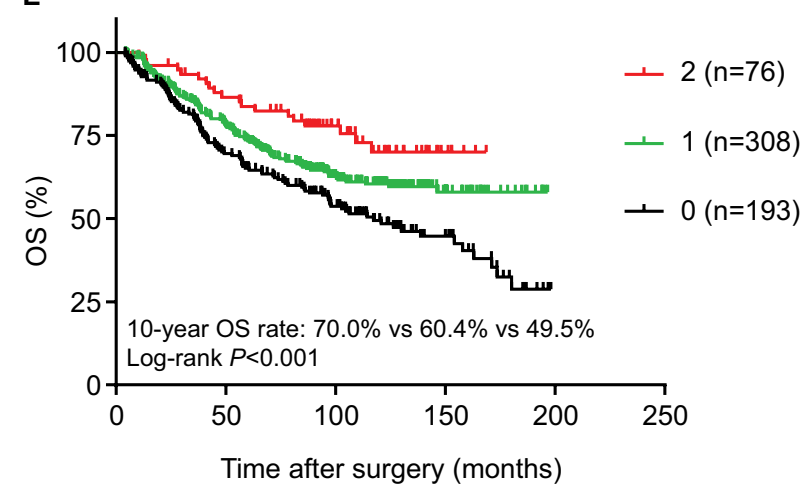

the low-LMR group (62.3\% vs 50.4\%, $P<0.001$; Figure $2 \mathrm{~A})$. The 10-year OS rate in the low-PLR group was significantly higher than that in the high-PLR group $(69.2 \%$ vs $55.8 \%$, $\mathrm{P}=0.008$; Figure 2B). Additionally, the 10 -year OS rate in the high-PNI group was significantly higher than that in the lowPNI group (61.8\% vs 50.1\%, $P=0.013$; Figure $2 \mathrm{C}$ ). However, no significant relationship was observed between NLR and prognosis in patients with stage IB NSCLC $(P=0.120$ for OS; Figure 2D). We performed the Kaplan-Meier analysis and

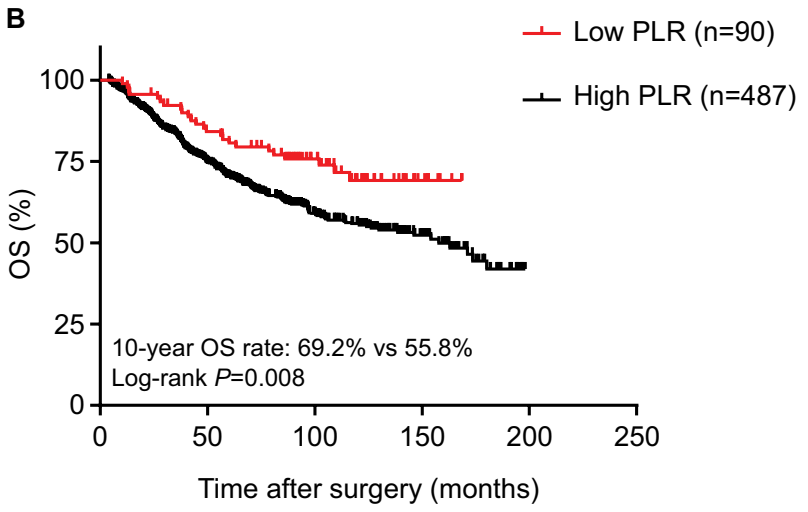

D

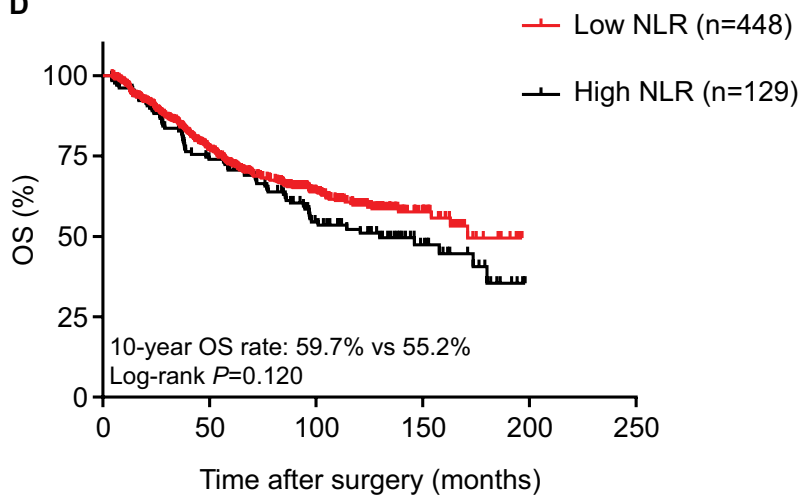

Figure 2 Kaplan-Meier curves of LMR (A), PLR (B), PNI (C), NLR (D), and LMR-PLR (E) for I0-year OS.

Abbreviations: LMR, lymphocyte-to-monocyte ratio; PLR, platelet-to-lymphocyte ratio; PNI, prognostic nutritional index; NLR, neutrophil-to-lymphocyte ratio; LMR-PLR, combination of preoperative LMR and PLR; OS, overall survival. 
log-rank test to determine survival differences among the three groups classified by the LMR-PLR score. The 10-year OS rates were $70.0 \%, 60.4 \%$, and $49.5 \%$, and the median survival times were 95.4, 87.8, and 83.2 months for LMRPLR $=2$, LMR-PLR $=1$, and LMR-PLR $=0$, respectively $(P<0.001$; Figure 2E). Further analyses were conducted in subgroups (adenocarcinoma and squamous carcinoma). We demonstrated that patients with LMR-PLR $=2$ displayed higher OS than those with LMR-PLR $=1$ or LMR-PLR $=0$ in the adenocarcinoma and squamous carcinoma subgroups (adenocarcinoma: $P<0.001$ for OS; Figure 3A; squamous carcinoma: $P=0.025$ for OS; Figure $3 \mathrm{~B}$ ). There was a significant difference between the 10-year OS values for ages $\leq 60$ and $>60$ years $(67.8 \%$ vs $46.8 \%, P<0.001)$. The 10 -year survival rate for patients without a history of smoking was significantly better than that of patients with a history of smoking $(63.4 \%$ vs $54.4 \%, P=0.032)$. We found that the 10 -year OS was significantly greater for RLN $\geq 12$ than for RLN $<12$ (62.4\% vs $52.6 \%, P=0.015)$.

\section{Discussion}

In this study, we investigated the prognostic value of clinicopathological variables (age, sex, smoking, cell types, tumor differentiation, visceral pleura invasion, bronchial invasion, postoperative chemotherapy, RLNs numbers, and RLNs stations) and inflammation-based factors (NLR, LMR, PLR, PNI, and LMR-PLR) in patients with stage IB NSCLC following radical surgical treatment. Confirmed by the univariate analysis, in addition to age, smoking history, cell type, bronchial invasion, RLNs numbers, LMR, PLR, PNI, and LMR-PLR were significantly associated with OS, whereas in the multivariate analysis, only age, smoking history, cell type, RLNs numbers, and LMR-PLR remained independent

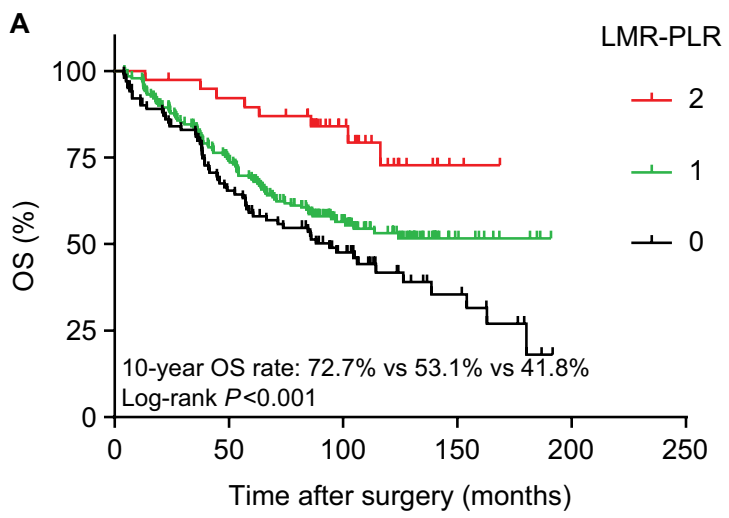

prognostic markers of OS. We found that preoperative LMRPLR was an independent positive prognostic factor in stage IB NSCLC.

Increasing evidence suggests that inflammatory cells are essential components of the microenvironment that play an important role in tumor progression. For nearly half a century, Coussens and Werb speculated that the occurrence and development of tumors were mediated by chronic inflammatory response. Inflammatory reactions are involved in the progression of tumors through a range of inflammatory cells, including lymphocytes, monocytes, platelets, and various signaling molecules in the cellular immune system. ${ }^{17}$ Epidemiological and clinical research reports support their view that $>15 \%$ of human cancers worldwide is caused by chronic inflammation. ${ }^{18}$ Research has shown that tumors have the ability to alter their host's systemic inflammation and immune response. As the tumor progresses, tumor promoting inflammation will overwhelm antitumor immunity. Inflammation is conducive to tumor cell division, proliferation, invasion, metastasis, angiogenesis, and suppression of antitumor adaptive immune responses. ${ }^{19,20}$

LMR is a measure of the relative difference between lymphocyte and monocyte counts and is an index of systemic inflammation. Lymphocytes and monocytes are two key components of the human immune system. The host's anticancer immune response relies mainly on lymphocytes, which activate the antitumor immune response processes of the host by releasing lymphatic factor to kill tumor cells. $^{21,22}$ It was discovered that $\mathrm{T}$ lymphocytes in animal models, such as $\mathrm{Ku}$ and Crowe, can lead to increased carcinogenicity in the head and neck and a reduction in the latency of the tumor. ${ }^{23}$ Monocytes can stimulate tumor cells or immune cells to produce a variety of chemokines,

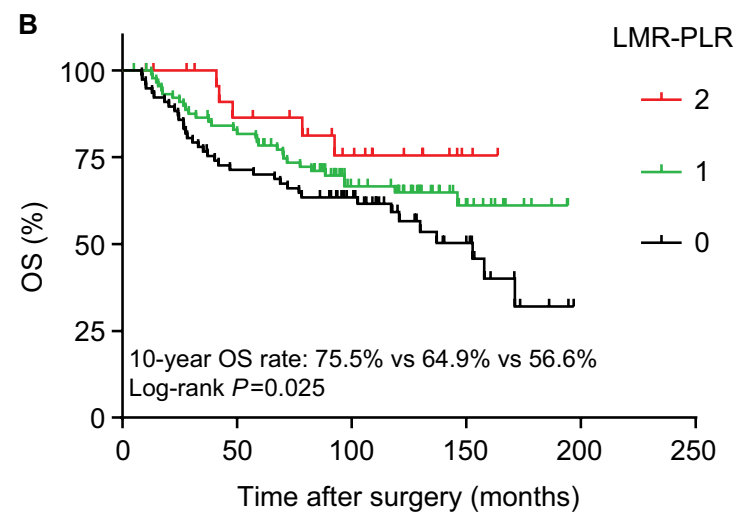

Figure 3 Survival curves of patients with adenocarcinoma or squamous carcinoma in LMR-PLR.

Note: OS curve of patients with $(\mathbf{A})$ adenocarcinoma with LMR-PLR $=2, \mathrm{LMR}-\mathrm{PLR}=\mathrm{I}$, and LMR-PLR $=0$ (log-rank test, $P<0.00 \mathrm{I})$ and $(\mathbf{B})$ squamous carcinoma with LMR-PLR $=2$, LMR-PLR $=1$, and LMR-PLR $=0$ (log-rank test, $P=0.025$ ).

Abbreviations: LMR, lymphocyte-to-monocyte ratio; PLR, platelet-to-lymphocyte ratio; LMR-PLR, combination of preoperative LMR and PLR; OS, overall survival. 
such as vascular endothelial growth factor, tumor necrosis factor alpha, and monocyte chemoattractant protein-1 to promote tumorigenesis, angiogenesis, and distant metastasis. ${ }^{24-27}$ Extensive studies have shown that tumor-associated macrophages (TAMs) originate from circulating monocyte precursors in blood. Tumor promoting TAMs orchestrate the tumor microenvironment by several mechanisms including regulating senescence, interacting with and contributing to extracellular matrix remodeling, promoting proliferation, progression, angiogenesis, and lymphangion genesis. ${ }^{28,29}$ This phenomenon explains why an elevated monocyte count leads to poor prognosis in patients with tumors. The prognostic value of LMR in lung cancer has already been investigated. The cutoff value of LMR in NSCLC varies from 3.09 to 4.56. ${ }^{30-32}$ By using the ROC curve, the cutoff value of LMR was determined to be 3.16 in our study, which is comparable with that in previous studies. ${ }^{30-32}$ Additionally, as dynamic reservoirs of various factors, platelets stimulate tumor cell proliferation and enhance angiogenesis by secreting a large number of cytokines and growth factors. Tumors, by secreting factors that retain platelets, protect the environment, thus positively affecting their survival. ${ }^{33}$ In addition, tumor cells can induce platelet aggregation, the so-called tumor cellinduced platelet aggregation, which allows for tumor cells to escape from immune surveillance and protect tumor cells from cytolysis. ${ }^{34}$ Similarly, elevated PLR has been correlated with poor survival in several malignancies. ${ }^{35,36}$ Furthermore, it is now indisputable that LMR and PLR provide information regarding the activity of tumor cells, and metastasis and invasion in patients, and that they reflect the degree of cancer progression. ${ }^{10,37}$ Although inflammation is complex, LMR and PLR are easily measurable biomarkers that reflect inflammation, and it has been shown that LMR and PLR are associated with prognosis in different types of carcinoma. ${ }^{10,38,39} \mathrm{~A}$ high LMR indicates an increased lymphocyte count and/ or a decreased monocyte count. Elevated lymphocytes can play a role in tumor defense by increasing the induction of cytotoxic cell death and inhibiting tumor cell proliferation and invasion. Recent studies have shown the effect of LMR on the prognosis of lung cancer patients. Go et al showed that low-LMR pretreatment is an independent, adverse prognostic indicator for predicting small-cell lung cancer survival. ${ }^{38}$ Our findings are consistent with previous studies on the relationship between LMR, PLR, and the prognosis of many other cancers, such as gastric cancer, pancreatic cancer, hepatocellular carcinoma, and breast cancer. ${ }^{40-45}$

NLR has been reported to be a useful biomarker to measure the inflammatory status of the immune system. ${ }^{46}$ The neutrophil is known to be able to aid the proliferation and survival of malignant cells, and promote angiogenesis and metastasis. Conversely, lymphocytes suppress tumor growth and invasion through their cytolytic activity. ${ }^{46}$ Taken together, patients with high NLR have relative lymphocytopenia and, as a result, may exhibit a poorer immune response to malignant advanced tumors, thereby worsening their prognosis. ${ }^{41,42}$ In this study, NLR was inconsistent in its results with other research reports. Among the inflammation-based scores examined, in addition to NLR, LMR and PLR were also based on lymphocyte count. Furthermore, PLR and PNI were defined as the combination of lymphocyte count and other factors. Therefore, there is a possibility that the prognostic power of NLR was offset.

PNI is based on serum albumin and lymphocyte. Low serum albumin level is correlated with malnutrition and weight loss and it is a risk factor for cancer mortality. NLR, LMR, PLR, and PNI are all based on peripheral lymphocyte counts. In the univariate analysis, low PNI was independently associated with poor OS in patients with stage IB NSCLC. However, we found no significant correlation between PNI and prognosis in the multivariate analysis, contrary to the findings of other studies. ${ }^{47,48}$ This is due to the fact that our study included various inflammation-related factors to evaluate the prognostic value in patients with stage IB NSCLC, which can better represent inflammatory conditions of patients.

In this study, we assessed the performance of preoperative NLR, LMR, PLR, and PNI in predicting the survival of patients with stage IB NSCLC. Among single factors, LMR is superior to PLR as a predictive factor for IB NSCLC. However, among combination factors, LMR-PLR was better than LMR alone or PLR alone.

In patients with stage IB NSCLC, cranial MRI and chest and abdomen enhancement $\mathrm{CT}$ scans can be used to assess the patient's clinical status and disease severity; however, these are both expensive and inconvenient. Preoperative LMR-PLR is poised as a convenient and inexpensive prognostic marker, as both LMR and PLR are widely used in conventional clinical practice. Routine blood tests are noninvasive and costeffective. Therefore, these ratios can be used as important indexes to predict disease prognosis. In this study, the loss rate for follow-up is low. We therefore believe that the results of this study are reliable.

However, there are some limitations in our research. First, we did not analyze other potential prognostic factors in this study. These factors should be included in future studies. Second, since only 577 patients were involved in the study, the sample volume was small, which could lead to a larger bias. Third, several disease conditions, such as ischemia and 
trauma, which may bias the blood circulating cell counts, were not taken into consideration.

\section{Conclusion}

The findings of our study indicate that the preoperative LMR-PLR score can be considered a valuable prognostic indicator in patients with stage IB NSCLC after surgery. A close relationship between LMR-PLR and cancer progression was also observed in patients with NSCLC who underwent surgery. Thus, LMR-PLR may be considered for routine clinical use as a reliable and low-cost biomarker.

\section{Acknowledgments}

We thank the department of follow-up for recording recurrence and death data in detail. Besides, we express our gratitude for support and suggestions in this study from staffs at the Department of Thoracic Surgery. The authors would like to thank the commissioners, Ming-Huang Hong and Chao-Nan Qian. The authenticity of this article has been validated by uploading the key raw data onto the Research Data Deposit public platform (http://www.researchdata.org. cn), with approval number RDDA2018000723.

\section{Disclosure}

The authors report no conflicts of interest in this work.

\section{References}

1. Siegel RL, Miller KD, Jemal A. Cancer statistics, 2018. CA Cancer J Clin. 2018;68(1):7-30.

2. Torre LA, Bray F, Siegel RL, Ferlay J, Lortet-Tieulent J, Jemal A. Global cancer statistics, 2012. CA Cancer J Clin. 2015;65(2):87-108.

3. Chen W, Zheng R, Baade PD, et al. Cancer statistics in China, 2015. CA Cancer J Clin. 2016;66(2):115-132.

4. Fan H, Shao ZY, Xiao YY, et al. Incidence and survival of non-small cell lung cancer in Shanghai: a population-based cohort study. $B M J$ Open. 2015;5(12):e9419.

5. Coleman MP, Forman D, Bryant H, et al. Cancer survival in Australia, Canada, Denmark, Norway, Sweden, and the UK, 1995-2007 (the International Cancer Benchmarking Partnership): an analysis of populationbased cancer registry data. Lancet. 2011;377(9760):127-138.

6. Harada M, Hato T, Horio H. Intratumoral lymphatic vessel involvement is an invasive indicator of completely resected pathologic stage I nonsmall cell lung cancer. J Thorac Oncol. 2011;6(1):48-54.

7. Hsu CP, Hsia JY, Chang GC, et al. Surgical-pathologic factors affect longterm outcomes in stage IB (pT2 N0 M0) non-small cell lung cancer: a heterogeneous disease. JThorac Cardiovasc Surg. 2009;138(2):426-433.

8. Liu W, Ha M, Yin N. Combination of platelet count and lymphocyte to monocyte ratio is a prognostic factor in patients undergoing surgery for non-small cell lung cancer. Oncotarget. 2017;8(42):73198-73207.

9. Shoji F, Morodomi Y, Akamine T, et al. Predictive impact for postoperative recurrence using the preoperative prognostic nutritional index in pathological stage I non-small cell lung cancer. Lung Cancer. 2016;98:15-21.

10. Song YJ, Wang LX, Hong YQ, et al. Lymphocyte to monocyte ratio is associated with response to first-line platinum-based chemotherapy and prognosis of early-stage non-small cell lung cancer patients. Tumour Biol. 2016;37(4):5285-5293.
11. Kacan T, Babacan NA, Seker M, et al. Could the neutrophil to lymphocyte ratio be a poor prognostic factor for non small cell lung cancers? Asian Pac J Cancer Prev. 2014;15(5):2089-2094.

12. Tomita M, Shimizu T, Ayabe T, Yonei A, Onitsuka T. Preoperative neutrophil to lymphocyte ratio as a prognostic predictor after curative resection for non-small cell lung cancer. Anticancer Res. 2011;31(9):2995-2998.

13. Zheng SH, Huang JL, Chen M, Wang BL, Ou QS, Huang SY. Diagnostic value of preoperative inflammatory markers in patients with glioma: a multicenter cohort study. J Neurosurg. 2018;129(3):583-592.

14. Xia H, Sun Z, Deng L, Zhu D, Wang D. Prognostic Significance of the Preoperative Lymphocyte to Monocyte Ratio in Patients With Stage I Non-Small Cell Lung Cancer Undergoing Complete Resection. Cancer Invest. 2016;34(8):378-384.

15. Tang H, Ma H, Peng F, et al. Prognostic performance of inflammationbased prognostic indices in locally advanced non-small-lung cancer treated with endostar and concurrent chemoradiotherapy. Mol Clin Oncol. 2016;4(5):801-806.

16. Neal CP, Cairns V, Jones MJ, et al. Prognostic performance of inflammation-based prognostic indices in patients with resectable colorectal liver metastases. Med Oncol. 2015;32(5):144.

17. Coussens LM, Werb Z. Inflammation and cancer. Nature. 2002;420(6917): $860-867$.

18. Ulich TR, del Castillo J, Keys M, Granger GA, Ni RX. Kinetics and mechanisms of recombinant human interleukin 1 and tumor necrosis factor-alpha-induced changes in circulating numbers of neutrophils and lymphocytes. J Immunol. 1987;139(10):3406-3415.

19. Hanahan D, Weinberg RA. Hallmarks of cancer: the next generation. Cell. 2011;144(5):646-674.

20. Hanahan D, Weinberg RA. The hallmarks of cancer. Cell. 2000;100(1):57-70.

21. Shibutani M, Maeda K, Nagahara H, et al. A high preoperative neutrophil-to-lymphocyte ratio is associated with poor survival in patients with colorectal cancer. Anticancer Res. 2013;33(8):3291-3294.

22. Shau HY, Kim A. Suppression of lymphokine-activated killer induction by neutrophils. J Immunol. 1988;141(12):4395-4402.

23. Ku TK, Crowe DL. Impaired T lymphocyte function increases tumorigenicity and decreases tumor latency in a mouse model of head and neck cancer. Int J Oncol. 2009;35(5):1211-1221.

24. Gordon S, Martinez FO. Alternative activation of macrophages: mechanism and functions. Immunity. 2010;32(5):593-604.

25. Heusinkveld M, de Vos van Steenwijk PJ, Goedemans R, et al. M2 macrophages induced by prostaglandin E2 and IL-6 from cervical carcinoma are switched to activated M1 macrophages by CD4+ Th1 cells. J Immunol. 2011;187(3):1157-1165.

26. Hefler L, Tempfer C, Heinze G, et al. Monocyte chemoattractant protein-1 serum levels in ovarian cancer patients. Br J Cancer. 1999;81(5): $855-859$.

27. Coussens LM, Werb Z. Inflammation and cancer. Nature 2002;420(6917):860-867.

28. Clear AJ, Lee AM, Calaminici M, et al. Increased angiogenic sprouting in poor prognosis $\mathrm{FL}$ is associated with elevated numbers of CD163+ macrophages within the immediate sprouting microenvironment. Blood. 2010;115(24):5053-5056.

29. Lin EY, Li JF, Gnatovskiy L, et al. Macrophages regulate the angiogenic switch in a mouse model of breast cancer. Cancer Res. 2006;66(23):11238-11246.

30. Song YJ, Wang LX, Hong YQ, et al. Lymphocyte to monocyte ratio is associated with response to first-line platinum-based chemotherapy and prognosis of early-stage non-small cell lung cancer patients. Tumour Biol. 2016;37(4):5285-5293.

31. Chen YM, Lai CH, Chang HC, et al. Baseline and Trend of Lymphocyteto-Monocyte Ratio as Prognostic Factors in Epidermal Growth Factor Receptor Mutant Non-Small Cell Lung Cancer Patients Treated with First-Line Epidermal Growth Factor Receptor Tyrosine Kinase Inhibitors. PLoS One. 2015;10(8):e136252. 
32. Lin GN, Peng JW, Xiao JJ, Liu DY, Xia ZJ. Prognostic impact of circulating monocytes and lymphocyte-to-monocyte ratio on previously untreated metastatic non-small cell lung cancer patients receiving platinum-based doublet. Med Oncol. 2014;31(7):70.

33. Sharma D, Brummel-Ziedins KE, Bouchard BA, Holmes CE. Platelets in tumor progression: a host factor that offers multiple potential targets in the treatment of cancer. J Cell Physiol. 2014;229(8):1005-1015.

34. Jurasz P, Alonso-Escolano D, Radomski MW. Platelet - cancer interactions: mechanisms and pharmacology of tumour cell-induced platelet aggregation. Br J Pharmacol. 2004;143(7):819-826.

35. Lee YY, Choi CH, Sung CO, et al. Prognostic value of pre-treatment circulating monocyte count in patients with cervical cancer: comparison with SCC-Ag level. Gynecol Oncol. 2012;124(1):92-97.

36. Sasaki A, Iwashita Y, Shibata K, Matsumoto T, Ohta M, Kitano S. Prognostic value of preoperative peripheral blood monocyte count in patients with hepatocellular carcinoma. Surgery. 2006;139(6):755-764.

37. Liu W, Ha M, Yin N. Combination of platelet count and lymphocyte to monocyte ratio is a prognostic factor in patients undergoing surgery for non-small cell lung cancer. Oncotarget. 2017;8(42):73198-73207.

38. Go SI, Kim RB, Song HN, et al. Prognostic significance of the lymphocyte-to-monocyte ratio in patients with small cell lung cancer. Med Oncol. 2014;31(12):323.

39. Deng YX, Lin JZ, Peng JH, et al. Lymphocyte-to-monocyte ratio before chemoradiotherapy represents a prognostic predictor for locally advanced rectal cancer. Onco Targets Ther. 2017;10:5575-5583.

40. Shimada H, Takiguchi N, Kainuma O, et al. High preoperative neutrophil-lymphocyte ratio predicts poor survival in patients with gastric cancer. Gastric Cancer. 2010;13(3):170-176.
41. Stotz M, Gerger A, Eisner F, et al. Increased neutrophil-lymphocyte ratio is a poor prognostic factor in patients with primary operable and inoperable pancreatic cancer. Br J Cancer. 2013;109(2):416-421.

42. Asano Y, Kashiwagi S, Onoda N, et al. Predictive Value of Neutrophil/Lymphocyte Ratio for Efficacy of Preoperative Chemotherapy in Triple-Negative Breast Cancer. Ann Surg Oncol. 2016;23(4): 1104-1110.

43. Garcea G, Ladwa N, Neal CP, Metcalfe MS, Dennison AR, Berry DP. Preoperative neutrophil-to-lymphocyte ratio (NLR) is associated with reduced disease-free survival following curative resection of pancreatic adenocarcinoma. World J Surg. 2011;35(4):868-872.

44. Mano Y, Shirabe K, Yamashita Y, et al. Preoperative neutrophil-tolymphocyte ratio is a predictor of survival after hepatectomy for hepatocellular carcinoma: a retrospective analysis. Ann Surg. 2013;258(2): 301-305.

45. Azab B, Bhatt VR, Phookan J, et al. Usefulness of the neutrophil-tolymphocyte ratio in predicting short- and long-term mortality in breast cancer patients. Ann Surg Oncol. 2012;19(1):217-224.

46. Gu XB, Tian T, Tian XJ, Zhang XJ. Prognostic significance of neutrophilto-lymphocyte ratio in non-small cell lung cancer: a meta-analysis. Sci Rep. 2015;5:12493.

47. He X, Li JP, Liu XH, et al. Prognostic value of C-reactive protein/ albumin ratio in predicting overall survival of Chinese cervical cancer patients overall survival: comparison among various inflammation based factors. J Cancer. 2018;9(10):1877-1884.

48. Polterauer S, Grimm C, Tempfer C, et al. C-reactive protein is a prognostic parameter in patients with cervical cancer. Gynecol Oncol. 2007;107(1):114-117.
Cancer Management and Research

\section{Publish your work in this journal}

Cancer Management and Research is an international, peer-reviewed open access journal focusing on cancer research and the optimal use of preventative and integrated treatment interventions to achieve improved outcomes, enhanced survival and quality of life for the cancer patient. The manuscript management system is completely online and includes

\section{Dovepress}

a very quick and fair peer-review system, which is all easy to use. Visit http://www.dovepress.com/testimonials.php to read real quotes from published authors. 